\title{
Acute effects of systemic inflammation upon neurovascular unit and cerebrovascular function.
}

Gaia Brezzo ${ }^{1}$ (gaia.brezzo@ed.ac.uk),

Julie Simpson² (julie.simpson@sheffield.ac.uk),

Kamar E. Ameen-Ali ${ }^{3}$ (k.ameen-ali@sheffield.ac.uk),

Jason Berwick ${ }^{3}$ (j.berwick@sheffield.ac.uk),

Chris Martin ${ }^{3}$ (c.martin@sheffield.ac.uk)

${ }^{1}$ University of Edinburgh, Centre for Discovery Brain Sciences

2 The University of Sheffield, Sheffield Institute for Translational Neuroscience (SITraN)

${ }^{3}$ The University of Sheffield, Department of Psychology,

\section{Corresponding author:}

Dr Gaia Brezzo

Centre for Discovery Brain Sciences

University of Edinburgh

Chancellor's Building

49 Little France Crescent

Edinburgh, EH16 4SB

gaia.brezzo@ed.ac.uk

Tel. +44 (0) 1312429494 


\section{Abstract}

\section{Background}

3 Brain health relies on a tightly regulated system known as neurovascular function whereby the

4 cellular constituents of the neurovascular unit (NVU) regulate cerebral haemodynamics in

5 accordance with neuronal metabolic demand. Disruption of neurovascular function impairs

6 brain health and is associated with the development of disease, including Alzheimer's disease

7 (AD). The NVU is the site of action of neuroinflammatory responses and contributes to the

8 transition of systemic inflammation to neuroinflammatory processes. Thus, systemic

9 inflammatory challenges may cause a shift in the NVU focus, prioritising neuroimmune over

10 neurovascular actions leading to altered neurovascular function.

\section{Methods}

12 Rats were injected with lipopolysaccharide (LPS) (2mg/kg) or vehicle and haemodynamic

13 responses to sensory and non-sensory (hypercapnia) stimuli were assessed in vivo. Following

14 imaging, animals were perfused and their brain extracted to histologically characterise

15 components of the NVU to determine the association between underlying pathology to altered

16 blood flow regulation in vivo.

\section{Results}

18 LPS-treated animals showed altered haemodynamic function and cerebrovascular dynamics 6

19 hours after LPS administration. Histological assessment identified a significant increase in

20 astrogliosis, microgliosis and endothelial activation in LPS-treated animals.

\section{Conclusions}

22 Our data shows that an acutely induced systemic inflammatory response is able to rapidly alter

23 in-vivo haemodynamic function and is associated with significant changes in the cellular

24 constituents of the NVU. We suggest that these effects are initially mediated by endothelial cells, 
bioRxiv preprint doi: https://doi.org/10.1101/498089; this version posted December 17, 2018. The copyright holder for this preprint (which was not certified by peer review) is the author/funder. All rights reserved. No reuse allowed without permission.

25 which are directly exposed to the circulating inflammatory stimulus and have been implicated

26 in regulating functional hyperaemia.

\section{$27 \quad$ Keywords}

28 Inflammation - lipopolysaccharide - neurovascular function - neurovascular unit 


\section{1. 1 Background}

30 Brain health and function are dependent on an extensively regulated blood supply. The local

31 regulation of blood flow in accordance with metabolic neuronal demands in the tissue is termed

32 neurovascular coupling and is orchestrated by the resident cells of the neurovascular unit

33 (NVU). Disruption to neurovascular coupling impairs the delivery of critical substrates to brain

34 cells and impedes the removal of by-products accumulated during cerebral metabolism [1].

35 Alterations of brain microenvironment and cellular interactions of the NVU have been

36 implicated in the development of a number of neurodegenerative diseases, including

37 Alzheimer's disease (AD) [2-4]. Nevertheless the process or processes by which neurovascular

38 function affects and is affected by neurodegenerative diseases in vivo, as well as the cellular

39 substrates of these effects, remain unclear.

40 Neurovascular coupling underpins the physiological basis of non-invasive functional

41 neuroimaging techniques, including functional magnetic resonance imaging (fMRI) and positron

42 emission tomography (PET) in which changes to brain blood flow and oxygenation are tracked

43 as surrogate markers for neuronal activity. Such neuroimaging techniques may provide new

opportunities to predict, detect, diagnose and study brain disease processes using non-invasive

45 imaging biomarkers, but are dependent on the mapping of relevant in vivo measurements to

46 neuropathological changes in the context of specific disease processes such as inflammation.

47 Mounting evidence highlights inflammation as a major factor in the development of many

48 neurodegenerative diseases, including AD [5-8]. Further evidence pinpoints inflammation as a

49 driver of neuropathology [9] and it has been shown to precede the development of amyloid-beta

$50(A \beta)$ plaques [10]. The NVU is the site of action of neuroinflammatory responses and

51 contributes to the transition of systemic inflammation to neuroinflammatory processes. Several

52 non-neuronal cells within the NVU are key players in the initiation and regulation of brain

53 inflammatory responses, as well as in mediating the effects of systemic inflammation upon brain

54 function. Activated astrocytes and microglia release a range of pro-inflammatory molecules [11- 
55 14]. Endothelial cells (ECs) also play an important role through upregulation of intercellular

56 adhesion molecules (ICAM-1) and vascular cellular adhesion molecules (VCAM-1) [15].

57 Research has also highlighted a beneficial role for inflammation, suggesting that directing the

58 inflammatory response may be of more therapeutic benefit than suppressing it [16, 17]. Glial

59 cells have been shown to have a neuroprotective role in the neuroinflammatory response [18-

$6021]$, highlighting the complexity and difficulty in pinpointing the roles and factors involved in

61 the pathophysiological cascade of inflammation.

62 To help elucidate the changes that occur in the context of inflammation, numerous models have

63 been developed. The peripheral lipopolysaccharide (LPS) injection method is a standard

64 technique of inducing inflammation both in vivo [22, 23] and in vitro [24]. Depending on dosage,

65 LPS treated animals display behavioural as well as cellular brain changes, predominately

66 associated with glial activation $[25,26]$. The current study investigated how acute systemic

67 inflammation impacts upon in vivo cerebrovascular function and the status of the underlying

68 NVU cells. This was investigated with a complementary set of in vivo neuroimaging measures in

69 a rat model, paired with detailed characterisation of the cellular pathology of the NVU with

70 immunohistochemistry methods.

\section{2. Methods}

\section{$72 \quad 2.1$ Animals and pharmacological treatment}

73 Female Hooded Lister rats (3-4 month old, 220g-320g) kept at a 12-hour light/dark cycle

74 environment at a temperature of $22{ }^{\circ} \mathrm{C}$ with access to food and water ad libitum were housed in

75 polycarbonate cages ( $n=3$ per cage) in the Biological Services Unit at the University of Sheffield.

76 Animals were fed conventional laboratory rat food. Sixteen animals were assigned to one of two

77 groups (control $n=8$ or LPS, $n=8$ ). Haemodynamic data were concurrently acquired in all

78 treatment groups at two time intervals ( 4 and 6 hours) after injection, to characterise the

79 development of the acutely induced LPS inflammatory response. 
80 Each animal received an intraperitoneal injection based on condition. Control animals were

81 administered a saline vehicle (1ml/kg), LPS animals received a dose of $2 \mathrm{mg} / \mathrm{kg}$ LPS-EB

82 (lipopolysaccharide from E.coli, 0111:B4 stain-TLR4 ligand, InvivoGen, Europe) dissolved in

83 endotoxin-free water (InvivoGen, Europe), following loss of consciousness from anaesthesia.

\section{$84 \quad 2.2$ Surgical procedures}

85 Details of surgical and experimental paradigms were similar to those reported in previous

86 publications from this laboratory [27-29]. Briefly, rats were anaesthetised with an

87 intraperitoneal injection (i.p) of urethane $(1.25 \mathrm{mg} / \mathrm{kg}$ in $25 \%$ solution), with additional doses of

88 anaesthetic $(0.1 \mathrm{ml})$ administered if necessary. Choice of anaesthetic was determined by

89 urethane's suitability for invasive surgery as well as long-lasting stability, which is essential in

90 experiments where data collection lasts several hours [30]. Anaesthetic depth was determined

91 by means of hindpaw pinch-reflex testing. Animals were tracheotomised to allow artificial

92 ventilation and regulation of respiratory parameters. A left femoral artery cannulation was

93 performed for mean arterial blood pressure (MABP) monitoring and blood gas analysis. A left

94 femoral vein cannulation was also performed to allow continuous administration of

95 phenylephrine in order to maintain blood pressure within a healthy physiological range (100-

$96110 \mathrm{mmHg}$ [31].

97 To enable haemodynamic recordings, the skull was exposed via a midline incision and a section

98 overlaying the left somatosensory cortex (barrel cortex) was thinned to translucency with a

99 dental drill. This section was located $1-4 \mathrm{~mm}$ posterior and 4-8mm lateral to Bregma [32]. A

100 thinned skull was typically $100-200 \mu \mathrm{m}$ thick with the local vasculature clearly observable. Care

101 was taken during thinning to ensure that the skull remained cool by frequently bathing the area

102 with saline.

103

104

105

\subsection{Physiological monitoring}

Temperature was maintained at $37{ }^{\circ} \mathrm{C}\left( \pm 0.5^{\circ} \mathrm{C}\right)$ throughout surgical and experimental procedures with the use of a homoeothermic blanket and rectal temperature probe (Harvard 
106 Apparatus, USA). Animals were artificially ventilated with room air using a small animal

107 ventilator (SAR 830, CWE Inc, USA); the breathing rate of each animal was assessed and

108 modified according to each individual animal's blood gas measurements. Respiration rates of

109 the animals ranged from 68 to 74 breaths per minute.

110 Blood pressure was monitored during the experiment with a pressure transducer (Wockhardt,

111 UK, 50 units of heparin per mL). Arterial blood from the femoral artery was allowed to flow

112 back from the cannula into a cartridge (iSTAT CG4+, Abbott Point of Care Inc., USA) and

113 analysed for blood gas to ensure normoxia and normocapnia using a blood gas analyser

114 (VetScan, iSTAT-1, Abaxis, USA). Physiological parameters were within normal ranges

throughout the experiment (mean values: $\mathrm{PO}_{2}=80 \mathrm{mmHg}( \pm 9.1) \mathrm{PCO}_{2}=30.4 \mathrm{mmHg}( \pm 3.7) \mathrm{SO}_{2}$

$=96 \%( \pm 1.2))$ Total volume of arterial blood extracted at one time did not surpass $95 \mu \mathrm{L}$.

117 Phenylephrine (Sigma, Aldrich) was administered into the left femoral vein using a syringe

pump (Sp200i, World Precision Instruments Inc., USA) to counteract reduced blood pressure

caused by anaesthesia. Dose was adjusted accordingly to the blood pressure of each individual

animal but remained in the range of $0.2-0.8 \mathrm{mg} / \mathrm{hr}$.

\subsection{Imaging}

Cerebral blood flow (CBF) data were acquired through a laser speckle contrast imaging (LSCI) camera (Full field Laser Perfusion Imager (FLPI-2), Moor Instruments, UK) which was positioned above the thinned cranial window. Images were acquired at $25 \mathrm{~Hz}$ at a spatial resolution of approximately $10 \mu \mathrm{m} /$ pixel. A 70 second baseline data acquisition period was acquired to obtain a measure of baseline blood flow.

The two-dimensional optical imaging spectroscopy (2D-OIS) technique was used to estimate changes in oxygenated $\left(\mathrm{HbO}_{2}\right)$, deoxygenated $(\mathrm{HbR})$ and total $(\mathrm{HbT})$ haemoglobin concentration in the rat barrel cortex. This technique has been previously described in detail $[33,34]$. OIS data was collected at a frame rate of $8 \mathrm{~Hz}$. The spectral analysis was based on the path length scaling algorithm (PLSA) [33], which uses a modified Beer-Lambert Law with a path length correction 
132 factor. In our analysis, baseline haemoglobin concentration in the tissue was estimated to be

$133104 \mu \mathrm{m}$ based on previous measurements [35] and oxygen saturation estimated to be $50 \%$ when

134 breathing room air.

\section{$135 \quad 2.5$ Stimulation paradigms}

136 Stimulation of the whisker pad was delivered via two subdermal stainless steel needle

137 electrodes (12mmx0.3mm, Natus neurology Incorporated, USA) directly inserted into the

138 whisker pad which transmitted an electrical current (1.0 mA). This intensity has been shown to

139 evoke a robust haemodynamic response without altering physiological factors such as blood

140 pressure and heart rate. The whiskers were stimulated at one of six frequencies $(1,2,5,10,20 \&$

$14140 \mathrm{~Hz}$ ), for two seconds with a stimulus pulse width of $0.3 \mathrm{~ms}$. The order of stimulation

142 frequencies was pseudorandomised with 10 trials at each frequency and an inter-trial interval

143 (ISI) of 25s. The electrical current is generated by an independent amplifier (Isolated Stimulator

144 DS3, Digitimer Ltd., UK) which directly attaches to the electrodes. All stimulation paradigms

145 were carried out 4 and 6 hours after saline/LPS administration.

\section{$146 \quad 2.6$ Hypercapnia challenge}

147 A hypercapnia challenge is used as a measure of vascular reactivity which is independent of neuronal activity changes. During hypercapnia, a 10\% concentration of carbon dioxide in medical air (9L medical air, $1 \mathrm{~L} \mathrm{CO}_{2}$ ) was administered to the air supply tube of the ventilator. Thirty-second long challenges were repeated four times at intervals of 210 seconds in the absence of whisker stimulation. An interval of 210 seconds ensured that the animal's physiological parameters returned to baseline levels before delivering the next challenge. These challenges were performed following the two stimulation paradigms.

\subsection{Perfusion}

Rats ( $n=11$ ) were transcardially perfused eight hours after LPS/saline administration (following in vivo data collection) with saline $\left(0.9 \%\right.$ warmed to $\left.37^{\circ} \mathrm{C}\right)$ with the addition of heparin 
158 (PFA) 01.M pH 7.4 in PBS. Saline and fixative were administered through a pump (Masterflex

$159 \mathrm{~L} / \mathrm{S}$, Cole-Parmer Instrument Company, UK) at a rate of $34 \mathrm{ml} / \mathrm{hr}$. Brains were stored in PFA

160 overnight at $4{ }^{\circ} \mathrm{C}$ and subdissected in four regions and embedded in paraffin wax. Serial sections

$161(5 \mu \mathrm{m})$ were cut from the paraffin-embedded tissue.

162

\subsection{Immunohistochemistry}

Immunohistochemistry was performed using a standard avidin-biotin complex-horse radish peroxidase (ABC-HRP) method, and visualised with diaminobenzidine ([DAB], Vector Laboratories, UK). A summary of utilised primary antibodies and their conditions of use are shown in Table 1. Isotype and no primary antibody controls were included in every run and no specific immunoreactivity was observed.

Table 1. Antibody sources and experimental conditions

\begin{tabular}{ccccc}
\hline Antibody & Isotype & Dilution (time, temp) & Antigen retrieval & Supplier \\
\hline Anti-GFAP & Rabbit IgG & $1: 1000(1 \mathrm{hr} \mathrm{RT})$ & MW 10 min, $\mathrm{pH} 9$ & Dako, UK \\
Anti-IBA1 & Rabbit IgG & $1: 400(1 \mathrm{hr} \mathrm{RT})$ & MW 10 min, pH9 & Abcam, UK \\
ICAM-1 & Goat IgG & $1: 400(1 \mathrm{hr}$ RT $)$ & MW 10 min, TSC pH 6.5 & R\&D, UK
\end{tabular}

\subsection{Data Processing and Analysis}

\subsubsection{In vivo imaging data}

Data were processed in Matlab (2016a) using custom written code. Imaging data were spatially smoothed and then analysed using SPM [36], with regions of interest (ROIs) selected from a thresholded activation map and expected to include contributions from arterial, venous and parenchymal (capillary bed) compartments. Two animals, one from each group, were excluded from final analysis due to the absence of well-localised stimulus-evoked responses, thereby a total $n=14$ was used for final analyses ( $n=7$ for each group). ROI size was consistent across animals, for both $\operatorname{LSCI}(F(1,13)=0.06, p=.811)$ and OIS $(F(1,13)=1.54 p=.239)$ data. LSCI and OIS time series of haemodynamic changes for each stimulation trials were then extracted from the ROI. LSCI time series were down-sampled to $5 \mathrm{~Hz}$ (from $25 \mathrm{~Hz}$ ). Data from each stimulation trial were extracted and divided by the pre-stimulus baseline period (10s), to yield a measure of 
181 percentage change (fractional changes) in $\mathrm{CBF}, \mathrm{HbO}_{2}, \mathrm{HbR}$ and $\mathrm{HbT}$. Time series were averaged

182 across trials according to stimulation condition. Area under the curve (AUC) and maxima for

183 each response were calculated.

184 Statistical comparisons between groups using maxima and AUC response values were

185 performed using multivariate ANOVAs (MANOVAs) for each time point ( 4 and 6 hours). A $p$

186 value below .05 was considered to be a significant effect. Additional independent sample t-tests

187 were conducted to further probe differences between experimental conditions. All statistical

188 analyses were conducted using SPSS 23.

\subsubsection{Immunohistochemistry data}

190 All images were taken from the contralateral side of the thinned cranial window (right side), to ensure inflammatory effects were not surgery (thinned cranial window) dependent. Images were taken from the somatosensory cortex (SS) and hippocampal subfields of the dentate gyrus (DG), CA1 and CA3. In the SS cortex three adjacent belt transects from the outer cortex through to the white matter border were taken for each animal at x20 magnification (Nikon microscope). SS area coordinates for captured imaged were taken between -.40 to -1.80 from Bregma (B) [37]. In the hippocampus, random field images were taken for each animal in the DG, CA1 and CA3 subfields. Hippocampus area coordinates for captured images were between 3.30 to -5.30 from B [37]. All slides were imaged blind in a randomised order. Percentage GFAP, IBA-1 and ICAM-1 area immunoreactivity was quantified using Analysis ${ }^{\mathrm{D}}$ software. All slides were analysed blind in a randomised order. One saline animal was excluded from analysis due to infection. Statistical analyses (independent sample t-tests) were performed using SPSS 23. A $p$ value below .05 was deemed significant. 
205 To assess any effects of treatment upon baseline CBF the average perfusion value across a 30s

206 period prior to the onset of stimulation at the start and end of the experimental protocol was

207 calculated. A one-way ANOVA on CBF baselines at 4 hours $(F(1,13)=0.10 p=.754)$ and at 6 hours

$208(F(1,13)=0.59, p=.458)$ post treatment found no difference between groups.

\subsection{Acute LPS treatment alters cerebrovascular responses to whisker stimulation}

Multivariate analyses of variance (MANOVAs) were applied to $\mathrm{HbO}_{2}, \mathrm{HbR}, \mathrm{HbT}$ and $\mathrm{CBF}$ administration at 4 hours and at 6 hours post treatment. All cases $(n=14)$ were included in the analysis. At 6 hours post injection, LPS administration altered the profile of haemodynamic responses across the investigated stimulation frequency range with a significant interaction between stimulation frequency and (LPS or saline) treatment $(F(20,190)=2.31, p=.002$; Wilks' $\Lambda=.486$ ). There were also significant univariate interaction effects for each haemodynamic measure as reported in Table 2. Post hoc analysis of individual stimulation frequencies using independent t-tests (Figure $1 \mathrm{~A}$ ) was used to assess if any particular frequency was more salient in driving the above interaction effect. Results indicate a significant increase in $\mathrm{HbO}_{2}(p=.0496)$ and $\operatorname{HbR}(p=.022)$ response magnitude at $5 \mathrm{~Hz}$ following LPS treatment. A representative haemodynamic response profile at a 5Hz stimulation frequency is plotted in Figure $1 \mathrm{~B}$ for each measure.

At 4 hours post injection, response magnitudes also appear altered, and especially for the $5 \mathrm{~Hz}$ interaction between stimulation frequency and treatment $(F(20,190)=1.34, p=.156$; Wilks' $\Lambda=$ $.645)$.

227 At both time-points treatment condition by itself did not result in any significant effect on 228 response magnitudes $(4 \mathrm{hrs:} F(4,9)=0.33, p=.852$; Wilks' $\Lambda=.872$; $6 \mathrm{hrs:} F(4,9)=1.25, p=.357$; 
230 observed in LPS-treated animals, the interaction with stimulation frequency was key in driving

231 significant differences between groups.

232 At both time points there was a significant effect of stimulation frequency on haemodynamic

233 response magnitude (4hrs: $F(20,190)=7.042, p<.001$; Wilks' $\Lambda=.159$; 6hrs: $F(20,190)=6.41$,

$234 p<.001$; Wilks' $\Lambda=.181$ [Figure $4.1 \mathrm{~A}$ ]), indicating the range of stimulus inputs was effective in

235 driving responses over a dynamic range, with significant effects for each haemodynamic

236 response measure (Table 2).

237 Table 2. Summary of univariate statistics for haemodynamic responses to a mixed frequency $2 \mathrm{~s}$

238 stimulation paradigm.

\begin{tabular}{|c|c|c|c|c|c|}
\hline Time & Factor & & $d f$ & $F$ & $p$ \\
\hline \multirow{12}{*}{$\begin{array}{c}+4 \text { hours after } \\
\text { injection }\end{array}$} & \multirow{4}{*}{ Frequency } & $\mathrm{HbO}_{2}$ & 5,60 & 15.19 & $<.001$ \\
\hline & & $\mathrm{HbR}$ & 5,60 & 18.68 & $<.001$ \\
\hline & & $\mathrm{HbT}$ & 5,60 & 11.80 & $<.001$ \\
\hline & & $\mathrm{CBF}$ & 5,60 & 17.98 & $<.001$ \\
\hline & \multirow{4}{*}{$\begin{array}{l}\text { Frequency } \mathrm{x} \\
\text { Treatment }\end{array}$} & $\mathrm{HbO}_{2}$ & 5,60 & 0.76 & $=.581$ \\
\hline & & $\mathrm{HbR}$ & 5,60 & 1.45 & $=.219$ \\
\hline & & $\mathrm{HbT}$ & 5,60 & 0.54 & $=.743$ \\
\hline & & CBF & 5,60 & 0.23 & $=.949$ \\
\hline & \multirow{4}{*}{ Treatment } & $\mathrm{HbO}_{2}$ & 1,12 & 0.10 & $=.758$ \\
\hline & & $\mathrm{HbR}$ & 1,12 & 0.24 & $=.635$ \\
\hline & & $\mathrm{HbT}$ & 1,12 & 0.02 & $=.892$ \\
\hline & & $\mathrm{CBF}$ & 1,12 & 0.15 & $=.703$ \\
\hline \multirow{12}{*}{$\begin{array}{l}+6 \text { hours after } \\
\text { injection }\end{array}$} & \multirow{4}{*}{ Frequency } & $\mathrm{HbO}_{2}$ & 5,60 & 21.45 & $<.001$ \\
\hline & & $\mathrm{HbR}$ & 5,60 & 23.86 & $<.001$ \\
\hline & & $\mathrm{HbT}$ & 5,60 & 15.25 & $<.001$ \\
\hline & & $\mathrm{CBF}$ & 5,60 & 19.13 & $<.001$ \\
\hline & \multirow{4}{*}{$\begin{array}{l}\text { Frequency } x \\
\text { Treatment }\end{array}$} & $\mathrm{HbO}$ & 5,60 & 6.48 & $<.001$ \\
\hline & & $\mathrm{HbR}$ & 5,60 & 7.92 & $<.001$ \\
\hline & & $\mathrm{HbT}$ & 5,60 & 4.75 & $=.001$ \\
\hline & & CBF & 5,60 & 2.64 & $=.032$ \\
\hline & \multirow{4}{*}{ Treatment } & $\mathrm{HbO}$ & 1,12 & 1.21 & $=.294$ \\
\hline & & $\mathrm{HbR}$ & 1,12 & 2.36 & $=.151$ \\
\hline & & $\mathrm{HbT}$ & 1,12 & 0.43 & $=.524$ \\
\hline & & $\mathrm{CBF}$ & 1,12 & 0.07 & $=.798$ \\
\hline
\end{tabular}


 \\ 3.3 LPS administration does not alter haemodynamic responses to hypercapnia}

240 Analysis of variance (MANOVA) were used to determine significant effects of treatment on

241 haemodynamic response magnitude (for $\mathrm{HbO}_{2}, \mathrm{HbR}, \mathrm{HbT}$ and $\mathrm{CBF}$ variables) to a $30 \mathrm{~s}$

242 hypercapnia challenge at 4 hours and 6 hours post treatment. Maxima (or minima for HbR)

243 values were used to quantify the response. Treatment had no significant effect on response

244 magnitude at 4 hours $(F(4,9)=1.42, p=.304$; Wilks' $\Lambda=.613)$ or at 6 hours $(F(4,9)=1.33, p=.331$;

245 Wilks' $\Lambda=.629$ ) (Figure 2).

3.4 LPS treated animals show a change in the cerebral metabolic rate of oxygen consumption

$247 \quad\left(\mathrm{CMRO}_{2}\right)$ following treatment

248 The method utilised to estimate $\mathrm{CMRO}_{2}$ was as described in $[28,38]$. Briefly the $\mathrm{CMRO}_{2}$ estimate

249 was calculated from $\mathrm{HbR}, \mathrm{HbT}$ and CBF values generated from OIS and LSCI data. $\mathrm{CMRO}_{2}$ in the

250 brain is directly linked to cellular energy consumption and neuronal activity [39], thus it can

251 provide a measure to assess the neurovascular coupling relationship by assessing changes in

252 oxygen delivery or oxygen metabolism. $\mathrm{CMRO}_{2}$ changes were estimated at both time points (4

253 hours and 6 hours) and for both saline and LPS treated animals (Figure 3). Response maxima

254 and AUC were calculated and analysed with one-way ANOVA. Whilst $\mathrm{CMRO}_{2}$ is estimated to

255 initially increase in saline and LPS treated animals, in both LPS conditions ( 4 hours and 6 hours)

256 there was a substantial decrease below baseline after the initial increase. There was no

257 significant difference in the response maxima between groups at 4 hours or 6 hours after

258 LPS/saline treatment ( 4 hours: $F(1,13)=3.5, p=.087$; 6 hours: $F(1,13)=2.5, p=.138)$. However a

259 one-way ANOVA on AUC reveals a significant difference at 6 hours $(F(1,13)=6.16, p=.029)$

260 between treatment groups. No AUC significant difference was found at 4 hours $(F(1,13)=2.05$,

$261 p=.178)$. 
263 GFAP immunolabelled the cell body and immediate processes of astrocytes in both cohorts

264 (Figure 4 A-D). Hypertrophic astrocytes were observed in LPS cases, indicative of a mild to

265 moderate astrogliosis phenotype. In LPS treated cohorts stronger immunoreactivity was

266 observed around visible vessels compared to controls in both SS and hippocampal regions. In

267 saline treated animals GFAP expression appeared to be predominately parenchymal

268 (Supplementary Figure 1 A-D). Levels of GFAP expression, assessed as percentage area

immunoreactivity showed a significant $74 \%$ increase in LPS cases in the SS cortex $(t(8)=-4.15$,

$p=.003$ ) (Figure 4 I). Within the hippocampal subfields immunoreactivity was similar to control

cases, no significant differences were found between LPS and saline treated groups (DG, $t(8)=-$

1.4, $p=.200(12 \%$ increase) (Figure $4 \mathrm{~J}), \mathrm{CA} 1, t(8)=-1.94, p=.089$ [38\% increase] or CA3, $t(8)=-$

$.34, p=.744[6 \%$ increase $])$.

IBA1 immunolabelled the cell body and ramified processes of microglia in both treatment

groups (Figure 4 E-H). Similarly to GFAP expression, LPS IBA1+ microglia showed stronger

immunoreactivity around vessels whereas, control cases displayed a predominant parenchymal

distribution (Supplementary Figure 1 E-H). LPS-treated animals displayed a hypertrophic

(Figure 4 F \& H). Microglial clustering was observed along vessels (Supplementary Figure 2).

Levels of IBA1 expression, assessed as percentage area immunoreactivity showed a significant

$109 \%$ increase in LPS treated animals in SS cortex $(t(8)=-3.84, p=.005)$ (Figure $4 \mathrm{~K})$ with more

282 intense immunolabelling of both the cell body and extending processes. In the hippocampus

$283 \mathrm{IBA}^{+}$microglia immunoreactivity for LPS treated was significantly increased in all examined

284 subfields (DG: $t(8)=-2.52, p=.036$ [72\% increase] (Figure 4 L), CA1: $t(8)=-4.09$ [119\% increase],

$285 p=.003$, CA3: $t(8)=-4.3, p=.003[153 \%$ increase $])$. 
288 ICAM-1 immunolabelled vessels (Figure 5 A-D) in both cohort groups although LPS treated

289 animals showed more intense immunoreactivity compared to controls. Furthermore,

290 immunolabelling of microglia was a feature of all LPS cases but was less intense than vessel

291 labelling. Percentage area assessment revealed a significant increase in ICAM-1 expression in

292 LPS cases with a 299\% area increase in the SS cortex $(t(8)=-6.5, p<.001$ ) (Figure $7 \mathrm{E}$ ) and in all

293 hippocampal subfields compared to controls (DG: $t(8)=-5.47, p=.001$ [236\% increase], (Figure 5

294 F) CA1: $t(8)=-4.73, p=.001[108 \%$ increase], CA3: $t(8)=-4.69, p=.002$ [186\% increase]).

\section{4. Discussion}

In this study we present evidence of an alteration of cerebrovascular function in animals acutely treated with LPS, demonstrated by changes in haemodynamic responses to a range of stimulus inputs. These findings have implications for the interpretation of neuroimaging data acquired in clinical and healthy cohorts where systemic inflammation may be present because physiological properties which underlie neuroimaging signals ( $\mathrm{CBF}, \mathrm{CBV}$ and $\mathrm{CMRO}_{2}$ ) acquired in humans may be altered. Moreover, characterisation of NVU cellular changes via immunohistochemistry showed morphological changes in astrocytes and microglia, alongside a marked increase in ICAM-1 expression, indicating ECs activation.

LPS administration significantly altered cerebrovascular function. These effects became evident at 6 hours after LPS administration, although similar (non-significant) trends could be observed at the 4 hour time point. It is difficult to assess why significant effects of altered cerebrovascular function are primarily driven by a specific stimulation frequency, although stimulation dependent effects have been previously reported in the literature $[40,41]$. Our results suggest the $5 \mathrm{~Hz}$ stimulation frequency to be particularly salient at driving this effect, and it has been previously reported that in anaesthetised animals this frequency is most effective in driving somatosensory neurovascular responses [41]. These results underline the complexity of the neurovascular coupling relationships, and the importance of including a dynamic range of 
313 sensory stimulations in neurovascular research to better assess how the neurovascular system

314 responds to different levels of input in health and under disease-related manipulations.

$315 \mathrm{CMRO}_{2}$ in the brain is considered to be an index of brain health and energy homeostasis [39] as

316 well as being directly linked to cell energy and neuronal activity [42]. Estimation of $\mathrm{CMRO}_{2}$ can

317 thus provide a measure to assess the neurovascular dynamics by assessing possible changes in

318 oxygen delivery or oxidative metabolism. $\mathrm{CMRO}_{2}$ estimations suggest an alteration in how

319 oxygen delivery is matched to metabolic demand; where the increase or decrease of CBF

320 relative to oxygen consumption is altered by LPS treatment. This alteration may in turn reflect a

321 change in neurovascular coupling but this claim requires validation with a direct measure of

322 neuronal activity. Nevertheless altered $\mathrm{CMRO}_{2}$ estimates indicate a change in the relationship

323 between the $\mathrm{HbR}, \mathrm{HbT}$ and CBF components of the haemodynamic response under LPS

324 treatment, from which the $\mathrm{CMRO}_{2}$ estimate is made. This would in turn predict that BOLD

325 signals, which are also derived from $\mathrm{HbR}, \mathrm{HbT}$ and $\mathrm{CBF}$ changes will be different under LPS

326 treatment, therefore caution should be taken in interpreting neuroimaging signals acquired

327 from subjects with systemic inflammation present.

$328 \mathrm{GFAP}^{+}$astrocytes in LPS-treated animals were more prominent, displaying greater

329 immunoreactivity of processes and cell body. Furthermore immunoreactivity was also localised

330 around vessels, suggesting increased GFAP expression by astrocyte endfeet. This phenotype is

331 suggestive of mild to moderate astrogliosis [14]. A non-significant difference between GFAP+

332 immunoreactivity in the hippocampus of LPS treated and control animals may be explained by

333 the dense population of GFAP+ astrocytes in the hippocampus compared to cortical areas [43,

334 44]. Further quantification utilising other GFAP isoforms alongside additional astrocyte markers

335 (such as ALDH1L1, nestin, vimentin, EAAT1 and EAAT2) may help to fully elucidate the

336 astrocyte response triggered by an acute LPS challenge. Similarly, characterisation of microglia

337 revealed increases in IBA1 expression and a shift from a ramified 'resting' morphology to a

338 hypertrophic profile characterised by an amoeboid appearance as seen in reactive microglia

$339[12,45]$. Furthermore IBA- $1^{+}$microglia in LPS cases were observed to cluster around present 
340 vessels, a response possibly driven by the increase in ICAM-1 expression of the inflamed

341 endothelium, as induction of ICAM-1 has been shown to correlate with increased microglial

342 activation in acute inflammation [15].

343 ICAM-1 has an important role in cell-to-cell adhesion interactions and has low expression in

344 cerebral microvessels in normal physiological conditions [46]. Its upregulation on the luminal

345 surface of ECs is increased in the presence of pro-inflammatory mediators and has been linked

346 to blood brain barrier (BBB) permeability during acute inflammation [15, 47]. In this study, we

347 demonstrate increased ICAM-1 expression on the luminal surface of ECs, as well as microglia

348 processes in LPS treated animals. ECs have already been shown to change their phenotypes in

349 support of various phases of the inflammatory process $[48,49]$. Furthermore EC location, at the

350 interface between brain and blood, as well as their expression of TLR-4 receptors [50] (directly

351 activated by the LPS strain used), could pinpoint these cells as initiators of the inflammatory

352 response. Alterations in EC function could then propagate to the rest of the NVU cells, activating

353 astrocytes and microglia. In support of this, a recent paper developing a concurrent cell-type

354 specific isolation method [51], reported gene expression changes (upregulation of pro-

355 inflammatory cytokines, chemokines, cell adhesion molecules, including ICAM1) in vascular

356 endothelia in response to a peripheral LPS challenge. These results indicate that vascular ECs

357 may be central in the initiation and transmission of the LPS response from the periphery to the

358 CNS via cytokines, chemokines and extracellular remodelling [51].

359 ECs have also been implicated as key players in mediating neurovascular coupling in health

360 [52]. Retrograde dilation of pial vessels following sensory stimulation is blocked if EC signalling

361 is interrupted and disruption of ECs at the pial surface leads to a significant attenuation of the

362 haemodynamic response [52]. These findings could help explain the increases in haemodynamic

363 measures observed in this study, where such changes could be mediated by increased activity of

364 ECs at the pial surface. Lastly, as ECs are exposed to the bloodstream, both BBB penetrating and

365 non-penetrating substances (such as LPS) could act upon these cells to mediate changes in

366 neurovascular coupling [53]. Intravenous administration of an anti-inflammatory substance 
367 (rofecoxib) was shown to attenuate cortical haemodynamic responses to sensory stimulation

368 [54]. Rofecoxib inhibits production of prostaglandins (PGs), which are present in the cerebral

369 endothelium. Furthermore, ECs through production and release of PGs, are key modulators of

370 vascular tone and thereby can readily influence the haemodynamic response. As such it is

371 possible that administration of LPS could be exerting its inflammatory effects through ECs/PGs

372 increasing haemodynamic responses to sensory stimulation. Further investigation relating EC

373 involvement in mediating haemodynamic changes and neurovascular coupling in health will be

374 key in understanding EC roles in systemic inflammation and their impact upon neurovascular

375 function and neurovascular coupling.

376 CNS cells including microglia [55] and astrocytes [56] possess TLR-4 receptors and thus should

377 be directly activated by an LPS challenge. However, lipopolysaccharides and pro-inflammatory

378 molecules are large and thus should have limited BBB permeability [57]. Evidence from

379 research radioactively labelling LPS $[58,59]$ has shown little LPS penetration of the BBB

$380(0.025 \%$ of the administered dose $)$ and this level of penetration was only present at doses of

$3813 \mathrm{mg} / \mathrm{kg}$ or higher. As such, in our model, we do not anticipate extravasation of LPS into the

382 parenchyma. This physical limitation suggests that the observable brain inflammatory response

383 produced by a peripheral administration of LPS is most likely mediated and initiated by ECs

384 (which also have TLR-4 receptors, as previously discussed) and may also be mediated by

alternative routes of communication between the brain and the periphery as opposed to a direct effect on glial TLR-4 brain receptors (for a comprehensive review see Holmes, 2013 [57]). This could explain why studies comparing brain and peripheral inflammatory challenges such as the one by Montacute et al. [60] report similar levels of brain inflammation in both challenges.

There is a pressing need to understand the communication pathways between the peripheral and central immune systems in order to understand the role of inflammation in neurological diseases and ageing and for the development of effective interventions combating inflammation. Neurodegenerative disease such as $\mathrm{AD}$ are characterised by a chronic low-grade inflammatory response, therefore a limitation of this study is the acute administration of an inflammatory 
394 challenge. It is, however, important to remember that as chronic and acute inflammation are not 395 completely separate processes and share some of the same mediators, including EC activation

396 [48], NVU changes measured in either acute or chronic inflammatory states can inform upon the 397 other. Thereby acute alterations in in vivo neurovascular function, which arise from NVU

398 cellular changes may also underpin the neurovascular changes observed in a chronic low-grade 399 inflammatory response. Ageing, is furthermore, a key factor in both AD pathology and in 400 inflammation. As such future work should aim to extend this model to include a low-dose 401 chronically treated group with different aged animals to maximise its relevance to human 402 pathology and human ageing.

403 Whilst we find no evidence of leukocyte extravasation in LPS treated cases, astrocytes and microglia are highly likely contributing to the neuroinflammatory response by secreting an array of cytokines. Future work should thus aim to characterise the inflammatory profile of glial cells in response to acute systemic inflammation. Lastly, varying species of LPS elicit different cytokines profiles, thereby producing different classes of immune response in vivo [61]. This is an important consideration for studies utilising LPS as a model of inflammation. The LPS strain utilised in this study, due to its ultrapure nature, only activates the TLR-4 pathway and thus offers a robust way of investigating the inflammatory-driven neurovascular and NVU effects

411 which are mediated by a specific pathway.

412 This study has implications for the understanding of how cerebrovascular function changes under an acute inflammatory response. Assessing neurovascular function across different stimulation frequencies enables a detailed assessment on short-latency neurovascular impulse-

415 response function $[29,41]$. This is of particular importance as human fMRI research studies rely 416 on short-latency impulse response functions to estimate the haemodynamic response [62]. It is

417 thus important to robustly assess haemodynamic responses in preclinical research to better 418 relate these findings to human fMRI studies and analysis. Furthermore the findings from the 419 current study are relevant to the application of fMRI in subjects or patients with a systemic 
420 inflammatory response, as they show that measures underlying fMRI signals $(\mathrm{CBF}, \mathrm{CMRO} 2$ and

421 CBV) may be altered in a systemic inflammatory state.

\section{5. Conclusion}

423 Our data shows that an acutely induced systemic inflammatory response is able to rapidly alter

424 in-vivo cerebrovascular function. Furthermore, it is associated with marked immunoreactivity

425 within the cellular constituents of the NVU. We suggest the inflammatory response to be initially

426 triggered by ECs as these cells are directly exposed to the bloodstream and have been

427 implicated in mediating neurovascular function in health. Functional changes in ECs may then

428 initiate a cascade of activation which propagates to other NVU cells such as glia. 


\section{List of abbreviations}

2D-OIS: Two-dimensional optical imaging spectroscopy

$\mathbf{A} \boldsymbol{\beta}$ : Amyloid-beta

AD: Alzheimer's disease

AQP4: Aquaporin 4

BBB: Blood brain barrier

BOLD: Blood oxygen level dependence

CBF: cerebral blood flow

DAB: 3,3'-Diaminobenzidine

DG: Dentate gyrus

fMRI: functional magnetic resonance imaging

GFAP: Glial fibrillary acidic protein

IBA1: Ionized calcium binding adaptor molecule 1

ICAM-1: intercellular adhesion molecule

IL-1: Interleukin 1

LPS: lipopolysaccharide

LSCI: Laser speckle contrast imaging

NVU: Neurovascular unit

VCAM-1: vascular cellular adhesion molecule

PET: Positron emission tomography

SS: Somatosensory cortex

TLR-4: Toll-like receptor 4

TNF- $\alpha$ : Tumour necrosis factor alpha 


\section{Declarations}

Ethics approval and consent to participate

The present study was approved by the UK Home Office under the Animals (Scientific

Procedures) Act 1986 and the University of Sheffield Animal Welfare and Ethical Review Body (AWERB, local ethics committee). All procedures were conducted under a U.K. Home office licence and have been reported in accordance with the ARRIVE guidelines.

Consent for publication

Not applicable

Availability of data and material

The datasets used and/or analysed during the current study available from the corresponding author on reasonable request.

\section{Competing interests}

The authors declare that there are no competing interests.

\section{Funding}

This study was supported by The Royal Society [CM, UF130327]; the Wellcome Trust [CM, WT093223AIA]; Alzheimer's Research UK [JB, KA-A, IRG2014-10] and The University of Sheffield [GB, PhD Teaching Fellowship].

Authors' contributions

GB, CM and JS designed the study. CM and JS supervised the project. GB collected and analysed in vivo and histology data. GB wrote the manuscript. GB, CM and JS interpreted the data. KA-A helped with initial histology data optimisation. All authors reviewed and edited the manuscript. All authors read and approved the final manuscript.

\section{Acknowledgements}

The authors would like to thank the Sheffield Institute for Translational Neuroscience (SITraN) histopathology technical team, in particular Lynne Baxter, for their work in preparation of immunohistological protocols and for providing technical assistance when required. 


\section{Figure legends}

Figure 1. Haemodynamic response to $2 \mathrm{~s}$ whisker stimulation at six frequencies at +4 and +6 hours after LPS/saline administration. (A) Bar charts show \% change at maxima or minima (HbR). Post hoc analysis on stimulation frequencies reveal significant effects at $5 \mathrm{~Hz}$ for $\mathrm{HbO}_{2}$ and $\mathrm{HbR}\left({ }^{*}\right.$ denotes significant differences at $\left.p<.05\right)$ between groups (B) Representative $5 \mathrm{~Hz}$ time series showing mean fractional changes. Grey rectangle indicates stimulation onset/offset and error bars indicate standard error of the mean.

\section{Figure $2 . \mathrm{HbO}_{2} \mathrm{HbR}, \mathrm{HbT}$ and $\mathrm{CBF}$ response to hypercapnia at $+4 \mathrm{hrs}$ and $+6 \mathrm{hrs}$ of} LPS/saline administration. (A) Time series show mean fractional changes and (B) bar charts show percentage change at maxima or minima. Grey rectangle indicates the $30 \mathrm{~s}_{2}$ challenge onset/offset. Error bars represent standard error of the mean.

Figure 3. $\mathrm{CMRO}_{2}$ estimation at $+4 \mathrm{hrs}$ and $+6 \mathrm{hrs}$ of $\mathrm{LPS} /$ saline administration. (A) Time series of estimated $\mathrm{CMRO}_{2}$ changes following whisker stimulation, (B) Bar chart showing percentage change at maxima and (C) Bar chart showing change in area under the curve (AUC, units are summed percentage change).

Figure 4. LPS-treatment changes in glial pathology. (A-D) GFAP immunolabelling of astrocyte cell body and immediate processes (E-H) IBA1 immunolabelling of microglia cell body and ramified processes. (I) GFAP SS percentage area immunoreactivity was significantly increased in LPS treated animals (74\% increase, $p=.003$ ) but (J) was not significantly different in DG ( $p=.200)$. IBA1 SS (K) and DG (L) percentage area immunoreactivity were significantly increased in LPS treated animals (SS: 109\% increase, $p=.005$; DG: $72 \%$ increase $p=.036$ ). Scale bar represents $50 \mu \mathrm{m}$ for $\mathbf{A}-\mathbf{H} .{ }^{* *}$ denotes $p<.010 *$ denotes $p<.05$.

Figure 5. LPS-treatment changes in ICAM-1 expression. (A-D) ICAM-1 immunolabelled vessels and (C-D) microglia processes. LPS-treatment led to increased percentage area ICAM-1 immunoreactivity in the SS cortex (E) (299\% increase, $p<.001)$ and in the DG (F) $(236 \%$ increase, $p=.001$ ). Scale bar represents $50 \mu \mathrm{m}$ for A-D. ${ }^{* *}$ denotes $p<.002$. 


\section{Supplementary Figure 1. Assessment of astrogliosis and microgliosis across brain}

regions. LPS treated cases displayed higher immunoreactivity on vessels (C-D) as well as a

parenchymal distribution. Astrocyte distribution in control cases is more parenchymal (A-B).

IBA-1+ microglia in control cases appear to be distributed primarily in the parenchyma (E-H)

whereas microglia in LPS cases display parenchymal localisation and are also more prominent around the vasculature (G-H). Scale bar represents $50 \mu \mathrm{m}$.

Supplementary Figure 2. IBA-1 vessel clustering. LPS treated cases displayed microglial clustering in cortex and hippocampus. Representative image from the CA1 hippocampal region. Scale bar represents $50 \mu \mathrm{m}$. 


\section{References}

1. Girouard H, ladecola C: Neurovascular coupling in the normal brain and in hypertension, stroke, and Alzheimer disease. J Appl Physiol (1985) 2006, 100:328335.

2. De Strooper B, Karran E: The cellular phase of Alzheimer's disease. Cell 2016, 164:603-615.

3. Sweeney MD, Sagare AP, Zlokovic BV: Cerebrospinal fluid biomarkers of neurovascular dysfunction in mild dementia and Alzheimer's disease. Journal of Cerebral Blood Flow \& Metabolism 2015, 35:1055-1068.

4. Zlokovic BV: Neurovascular pathways to neurodegeneration in Alzheimer's disease and other disorders. Nat Rev Neurosci 2011, 12:723-738.

5. Cunningham C: Microglia and neurodegeneration: the role of systemic inflammation. Glia 2013, 61:71-90.

6. Frank-Cannon TC, Alto LT, McAlpine FE, Tansey MG: Does neuroinflammation fan the flame in neurodegenerative diseases? Molecular neurodegeneration 2009, 4:1.

7. Gao H-M, Zhang F, Zhou H, Kam W, Wilson B, Hong J-S: Neuroinflammation and [alpha]-Synuclein Dysfunction Potentiate Each Other, Driving Chronic Progression of Neurodegeneration in a Mouse Model of Parkinson's Disease. Environmental health perspectives 2011, 119:807.

8. Heppner FL, Ransohoff RM, Becher B: Immune attack: the role of inflammation in Alzheimer disease. Nature Reviews Neuroscience 2015, 16:358-372.

9. Krstic D, Madhusudan A, Doehner J, Vogel P, Notter T, Imhof C, Manalastas A, Hilfiker M, Pfister S, Schwerdel C: Systemic immune challenges trigger and drive Alzheimerlike neuropathology in mice. Journal of neuroinflammation 2012, 9:1.

10. Wright AL, Zinn R, Hohensinn B, Konen LM, Beynon SB, Tan RP, Clark IA, Abdipranoto A, Vissel B: Neuroinflammation and neuronal loss precede $A \boldsymbol{\beta}$ plaque deposition in the hAPP-J20 mouse model of Alzheimer's disease. PLoS One 2013, 8:e59586.

11. Saijo K, Winner B, Carson CT, Collier JG, Boyer L, Rosenfeld MG, Gage FH, Glass CK: A Nurr1/CoREST pathway in microglia and astrocytes protects dopaminergic neurons from inflammation-induced death. Cell 2009, 137:47-59.

12. Boche D, Perry V, Nicoll J: Review: activation patterns of microglia and their identification in the human brain. Neuropathology and applied neurobiology 2013, 39:3-18.

13. Sofroniew MV: Molecular dissection of reactive astrogliosis and glial scar formation. Trends in neurosciences 2009, 32:638-647.

14. Sofroniew MV, Vinters HV: Astrocytes: biology and pathology. Acta neuropathologica 2010, 119:7-35.

15. Huber JD, Campos CR, Mark KS, Davis TP: Alterations in blood-brain barrier ICAM-1 expression and brain microglial activation after $\boldsymbol{\lambda}$-carrageenan-induced inflammatory pain. American Journal of Physiology-Heart and Circulatory Physiology 2006, 290:H732-H740.

16. Buckwalter MS, Wyss-Coray T: Modelling neuroinflammatory phenotypes in vivo. Journal of neuroinflammation 2004, 1:10.

17. Wyss-Coray T, Mucke L: Inflammation in neurodegenerative disease-a doubleedged sword. Neuron 2002, 35:419-432.

18. Morgan SC, Taylor DL, Pocock JM: Microglia release activators of neuronal proliferation mediated by activation of mitogen-activated protein kinase, phosphatidylinositol-3-kinase/Akt and delta-Notch signalling cascades. Journal of Neurochemistry 2004, 90:89-101.

19. Polazzi E, Gianni T, Contestabile A: Microglial cells protect cerebellar granule neurons from apoptosis: Evidence for reciprocal signaling. Glia 2001, 36:271-280. 
20. Bush TG, Puvanachandra N, Horner CH, Polito A, Ostenfeld T, Svendsen CN, Mucke L, Johnson MH, Sofroniew MV: Leukocyte infiltration, neuronal degeneration, and neurite outgrowth after ablation of scar-forming, reactive astrocytes in adult transgenic mice. Neuron 1999, 23:297-308.

21. Chen Y, Vartiainen NE, Ying W, Chan PH, Koistinaho J, Swanson RA: Astrocytes protect neurons from nitric oxide toxicity by a glutathione? dependent mechanism. Journal of neurochemistry 2001, 77:1601-1610.

22. Hauss-Wegrzyniak B, Dobrzanski P, Stoehr JD, Wenk GL: Chronic neuroinflammation in rats reproduces components of the neurobiology of Alzheimer's disease. Brain research 1998, 780:294-303.

23. Pintado C, Gavilán MP, Gavilán E, García-Cuervo L, Gutiérrez A, Vitorica J, Castaño A, Ríos RM, Ruano D: Lipopolysaccharide-induced neuroinflammation leads to the accumulation of ubiquitinated proteins and increases susceptibility to neurodegeneration induced by proteasome inhibition in rat hippocampus. Journal of neuroinflammation 2012, 9:1.

24. Lehnardt S, Massillon L, Follett P, Jensen FE, Ratan R, Rosenberg PA, Volpe JJ, Vartanian T: Activation of innate immunity in the CNS triggers neurodegeneration through a Toll-like receptor 4-dependent pathway. Proceedings of the National Academy of Sciences 2003, 100:8514-8519.

25. Nazem A, Sankowski R, Bacher M, Al-Abed Y: Rodent models of neuroinflammation for Alzheimer's disease. Journal of neuroinflammation 2015, 12:1.

26. Zakaria R, Yaacob WW, Othman Z, Long I, Ahmad A, Al-Rahbi B, Oman M: Lipopolysaccharide-induced memory impairment in rats: a model of Alzheimer's disease. Physiological research 2017.

27. Hewson-Stoate N, Jones M, Martindale J, Berwick J, Mayhew J: Further nonlinearities in neurovascular coupling in rodent barrel cortex. Neuroimage 2005, 24:565-574.

28. Jones M, Berwick J, Johnston D, Mayhew J: Concurrent optical imaging spectroscopy and laser-Doppler flowmetry: the relationship between blood flow, oxygenation, and volume in rodent barrel cortex. Neuroimage 2001, 13:1002-1015.

29. Martindale J, Mayhew J, Berwick J, Jones M, Martin C, Johnston D, Redgrave P, Zheng Y: The hemodynamic impulse response to a single neural event. J Cereb Blood Flow Metab 2003, 23:546-555.

30. Angel A, Gratton D, A The effect of anaesthetic agents on cerebral cortical responses in the rat. British journal of pharmacology 1982, 76:541-549.

31. Golanov E, Yamamoto S, Reis D: Spontaneous waves of cerebral blood flow associated with a pattern of electrocortical activity. American Journal of PhysiologyRegulatory, Integrative and Comparative Physiology 1994, 266:R204-R214.

32. Chapin JK, Lin C-S: Mapping the body representation in the SI cortex of anesthetized and awake rats. The Journal of Comparative Neurology 1984, 229:199-213.

33. Berwick J, Johnston D, Jones M, Martindale J, Redgrave P, McLoughlin N, Schiessl I, Mayhew J: Neurovascular coupling investigated with two?dimensional optical imaging spectroscopy in rat whisker barrel cortex. European Journal of Neuroscience 2005, 22:1655-1666.

34. Sharp PS, Shaw K, Boorman L, Harris S, Kennerley AJ, Azzouz M, Berwick J: Comparison of stimulus-evoked cerebral hemodynamics in the awake mouse and under a novel anesthetic regime. Scientific reports 2015, 5:12621.

35. Kennerley AJ, Berwick J, Martindale J, Johnston D, Papadakis N, Mayhew JE: Concurrent fMRI and optical measures for the investigation of the hemodynamic response function. Magnetic resonance in medicine 2005, 54:354-365.

36. Statistical Parametric Mapping (SPM) [http://www.fil.ion.ucl.ac.uk/spm/]

37. Paxinos G, Watson C: The Rat Brain in Stereotaxic Coordinates. Academic Press; 1998.

38. Mayhew J, Johnston D, Martindale J, Jones M, Berwick J, Zheng Y: Increased oxygen consumption following activation of brain: theoretical footnotes using spectroscopic data from barrel cortex. Neuroimage 2001, 13:975-987. 
39. Liu P, Huang H, Rollins N, Chalak LF, Jeon T, Halovanic C, Lu H: Quantitative assessment of global cerebral metabolic rate of oxygen (CMRO2) in neonates using MRI. NMR in Biomedicine 2014, 27:332-340.

40. Spain A, Howarth C, Khrapitchev AA, Sharp T, Sibson NR, Martin C: Neurovascular and neuroimaging effects of the hallucinogenic serotonin receptor agonist psilocin in the rat brain. Neuropharmacology 2015, 99:210-220.

41. Martin C, Martindale J, Berwick J, Mayhew J: Investigating neural-hemodynamic coupling and the hemodynamic response function in the awake rat. Neuroimage 2006, 32:33-48.

42. Ge Y, Zhang Z, Lu H, Tang L, Jaggi H, Herbert J, Babb JS, Rusinek H, Grossman RI: Characterizing brain oxygen metabolism in patients with multiple sclerosis with T2-relaxation-under-spin-tagging MRI. Journal of Cerebral Blood Flow \& Metabolism 2012, 32:403-412.

43. Gomi H, Yokoyama T, Fujimoto K, Ikeda T, Katoh A, Itoh T, Itohara S: Mice devoid of the glial fibrillary acidic protein develop normally and are susceptible to scrapie prions. Neuron 1995, 14:29-41.

44. Shibuki K, Gomi H, Chen L, Bao S, Kim JJ, Wakatsuki H, Fujisaki T, Fujimoto K, Katoh A, Ikeda T: Deficient cerebellar long-term depression, impaired eyeblink conditioning, and normal motor coordination in GFAP mutant mice. Neuron 1996, 16:587-599.

45. Sun J, Zheng JH, Zhao M, Lee S, Goldstein H: Increased in vivo activation of microglia and astrocytes in the brains of mice transgenic for an infectious R5 human immunodeficiency virus type 1 provirus and for CD4-specific expression of human cyclin $\mathbf{T} 1$ in response to stimulation by lipopolysaccharides. Journal of virology 2008, 82:5562-5572.

46. Wertheimer SJ, Myers CL, Wallace RW, Parks TP: Intercellular adhesion molecule-1 gene expression in human endothelial cells. Differential regulation by tumor necrosis factor-alpha and phorbol myristate acetate. Journal of Biological Chemistry 1992, 267:12030-12035.

47. da Fonseca ACC, Matias D, Garcia C, Amaral R, Geraldo LH, Freitas C, Lima FRS: The impact of microglial activation on blood-brain barrier in brain diseases. Frontiers in cellular neuroscience 2014, 8.

48. Pober JS, Sessa WC: Evolving functions of endothelial cells in inflammation. Nature Reviews Immunology 2007, 7:803-815.

49. Pober JS, Cotran RS: The role of endothelial cells in inflammation. Transplantation 1990, 50:537-544.

50. Verma S, Nakaoke R, Dohgu S, Banks WA: Release of cytokines by brain endothelial cells: a polarized response to lipopolysaccharide. Brain, behavior, and immunity 2006, 20:449-455.

51. Swartzlander DB, Propson NE, Roy ER, Saito T, Saido T, Wang B, Zheng H: Concurrent cell type-specific isolation and profiling of mouse brains in inflammation and Alzheimer's disease. JCI insight 2018, 3.

52. Chen BR, Kozberg MG, Bouchard MB, Shaik MA, Hillman EM: A critical role for the vascular endothelium in functional neurovascular coupling in the brain. Journal of the American Heart Association 2014, 3:e000787.

53. D'Esposito M, Deouell LY, Gazzaley A: Alterations in the BOLD fMRI signal with ageing and disease: a challenge for neuroimaging. Nature reviews Neuroscience 2003, 4:863.

54. Bakalova R, Matsuura T, Kanno I: The cyclooxygenase inhibitors indomethacin and Rofecoxib reduce regional cerebral blood flow evoked by somatosensory stimulation in rats. Experimental Biology and Medicine 2002, 227:465-473.

55. Marzolo MP, Von Bernhardi R, Bu G, Inestrosa NC: Expression of $\alpha 2$ macroglobulin receptor/low density lipoprotein receptor?related protein (LRP) in rat microglial cells. Journal of neuroscience research 2000, 60:401-411. 
56. Chakravarty S, Herkenham M: Toll-like receptor 4 on nonhematopoietic cells sustains CNS inflammation during endotoxemia, independent of systemic cytokines. Journal of Neuroscience 2005, 25:1788-1796.

57. Holmes C: Review: systemic inflammation and Alzheimer's disease. Neuropathology and applied neurobiology 2013, 39:51-68.

58. Banks WA, Gray AM, Erickson MA, Salameh TS, Damodarasamy M, Sheibani N, Meabon JS, Wing EE, Morofuji Y, Cook DG: Lipopolysaccharide-induced blood-brain barrier disruption: roles of cyclooxygenase, oxidative stress, neuroinflammation, and elements of the neurovascular unit. Journal of neuroinflammation 2015, 12:223.

59. Banks WA, Robinson SM: Minimal penetration of lipopolysaccharide across the murine blood-brain barrier. Brain, behavior, and immunity 2010, 24:102-109.

60. Montacute R, Foley K, Forman R, Else KJ, Cruickshank SM, Allan SM: Enhanced susceptibility of triple transgenic Alzheimer's disease (3xTg-AD) mice to acute infection. Journal of Neuroinflammation 2017, 14:50.

61. Pulendran B, Kumar P, Cutler CW, Mohamadzadeh M, Van Dyke T, Banchereau J: Lipopolysaccharides from distinct pathogens induce different classes of immune responses in vivo. The Journal of Immunology 2001, 167:5067-5076.

62. Logothetis NK: What we can do and what we cannot do with fMRI. Nature 2008, 453:869-878. 
bioRxiv preprint doi: https://doi.org/10.1101/498089; this version posted December 17, 2018. The copyright holder for this preprint (which was not certified by peer review) is the author/funder. All rights reserved. No reuse allowed without permission.

A
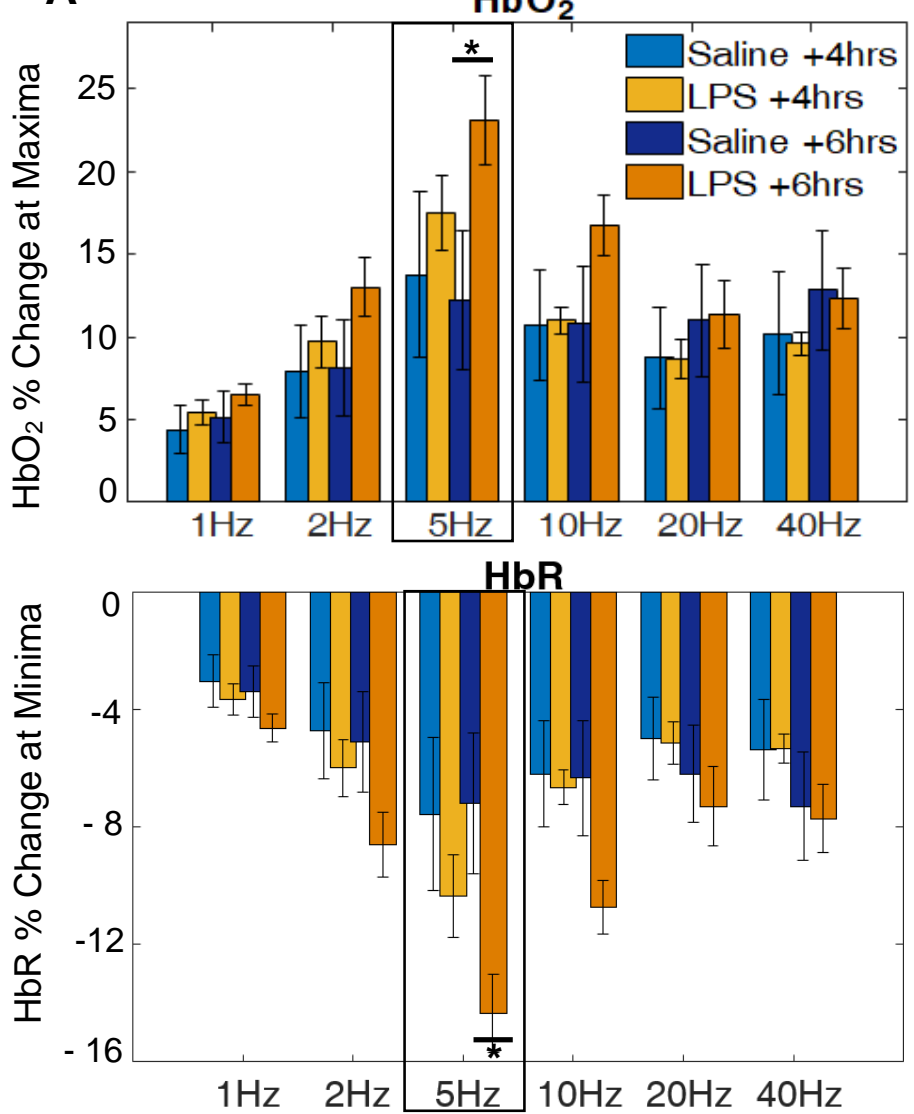

HbT
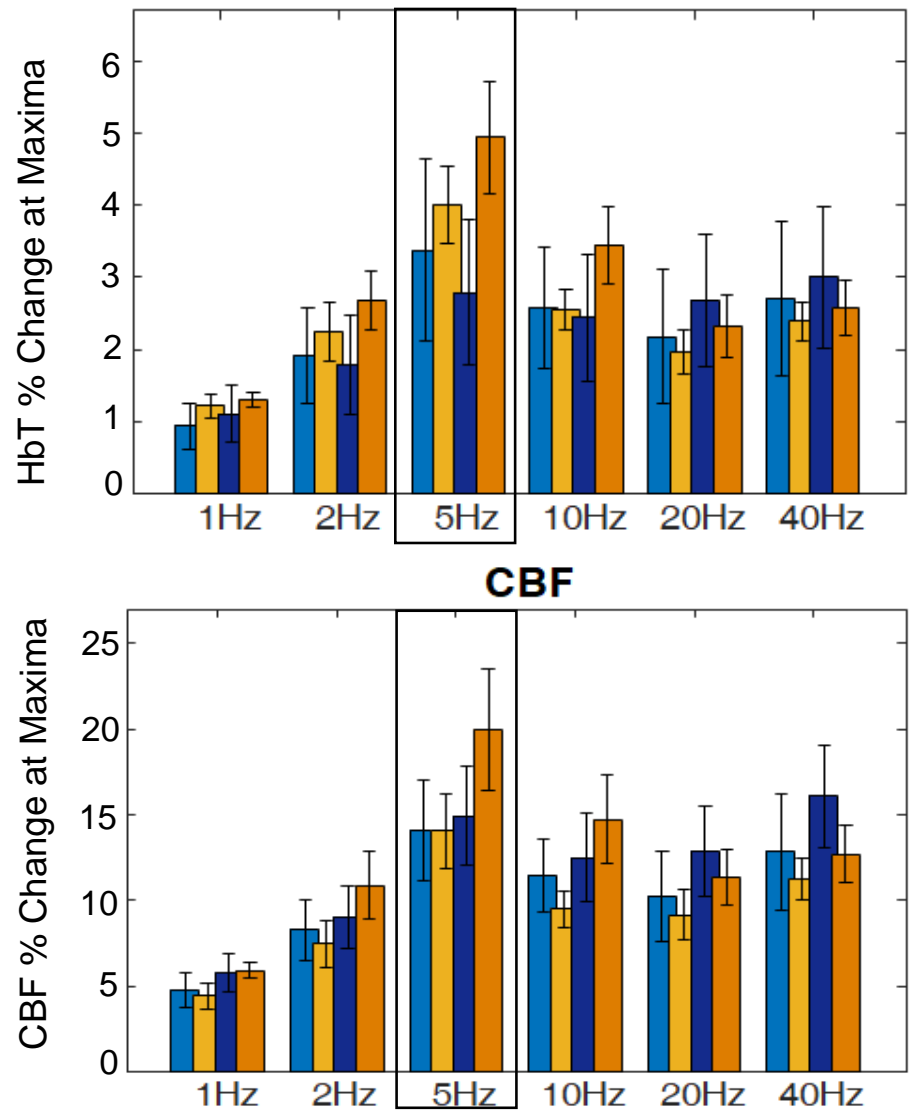

Frequency

\section{B}
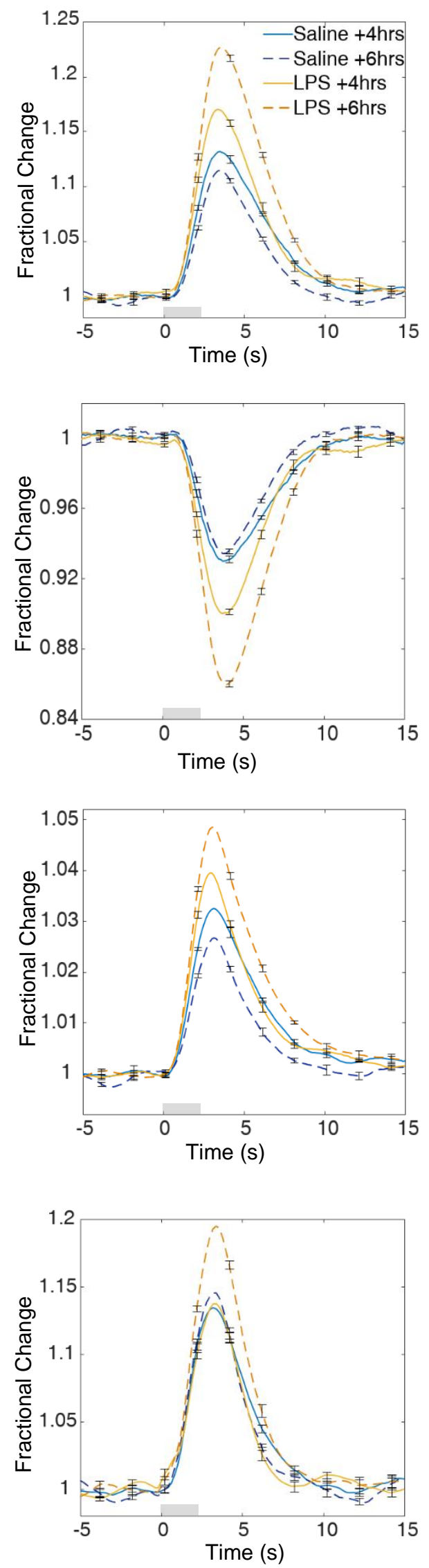

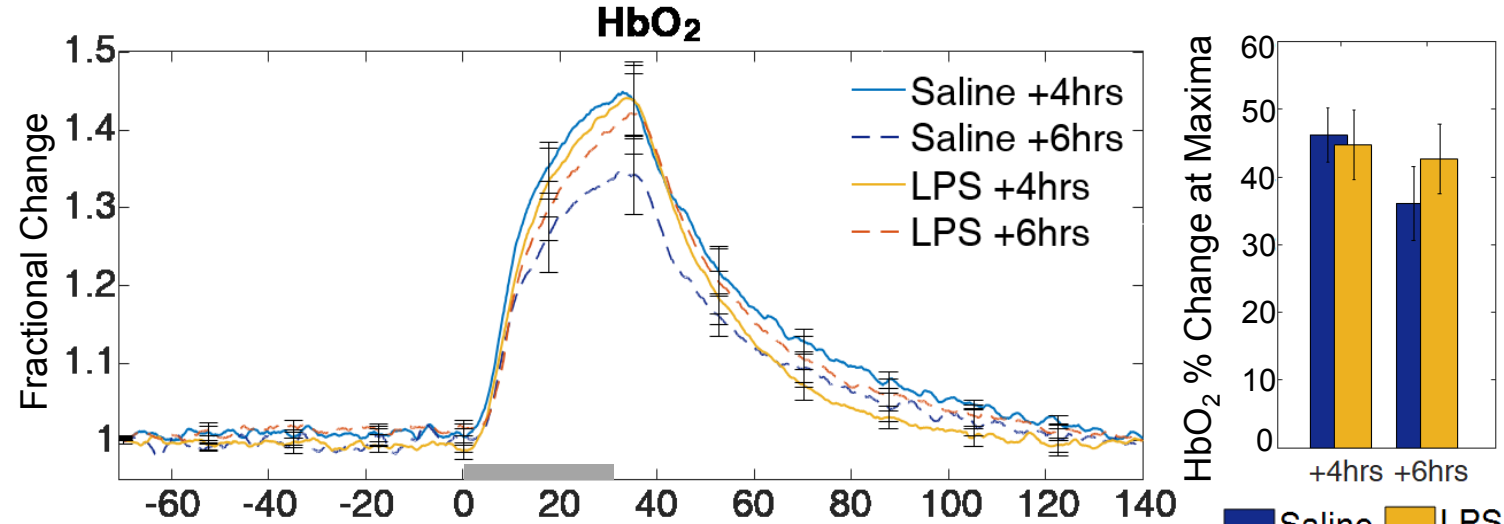

1.1 non
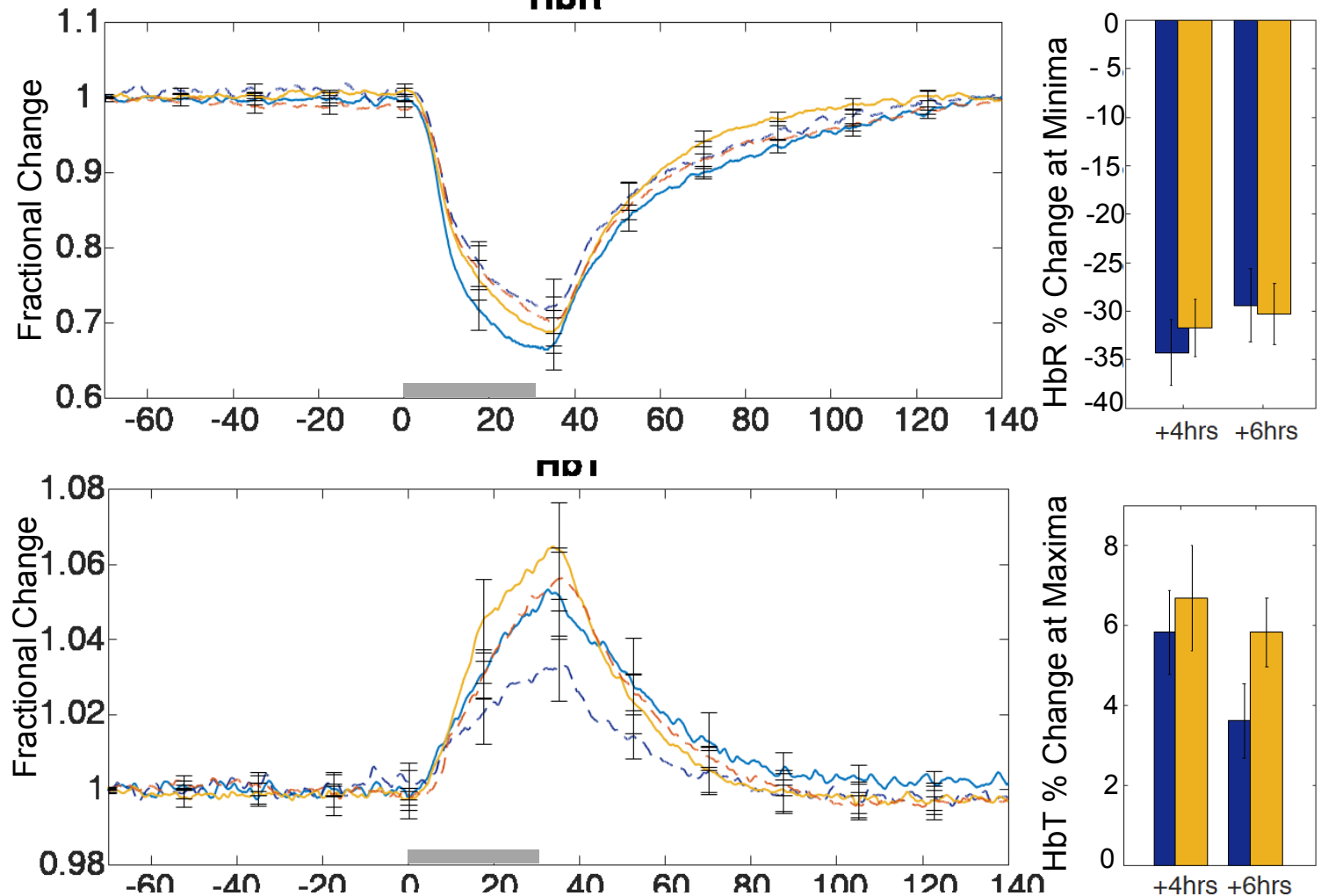

1.5

CBF

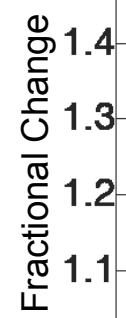

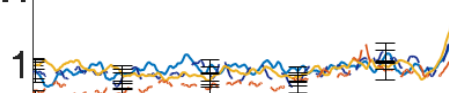
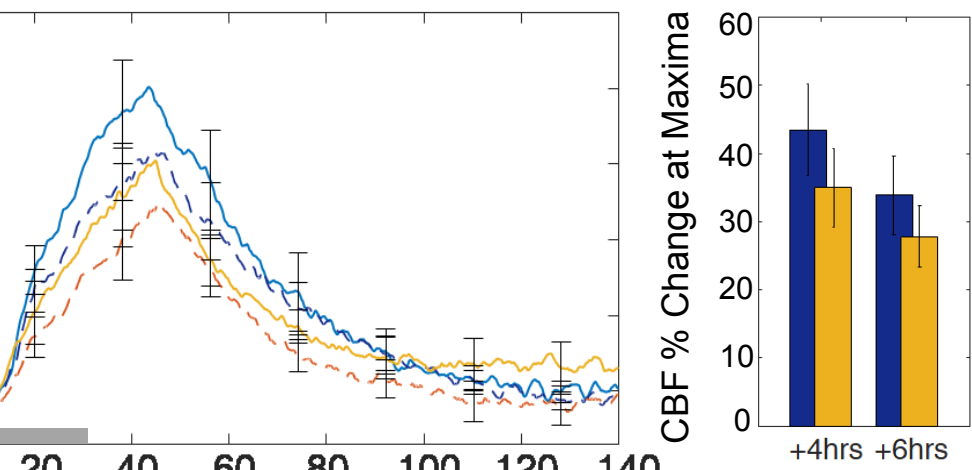

$$
\begin{aligned}
& \begin{array}{lllllllllll}
-60 & -40 & -20 & 0 & 20 & 40 & 60 & 80 & 100 & 120 & 140
\end{array} \\
& \text { Time (s) }
\end{aligned}
$$


bioRxiv preprint doi: https://doi.org/10.1101/498089; this version posted December 17, 2018. The copyright holder for this preprint (which was not certified by peer review) is the author/funder. All rights reserved. No reuse allowed without permission.

A

$\mathrm{CMRO}_{2}$
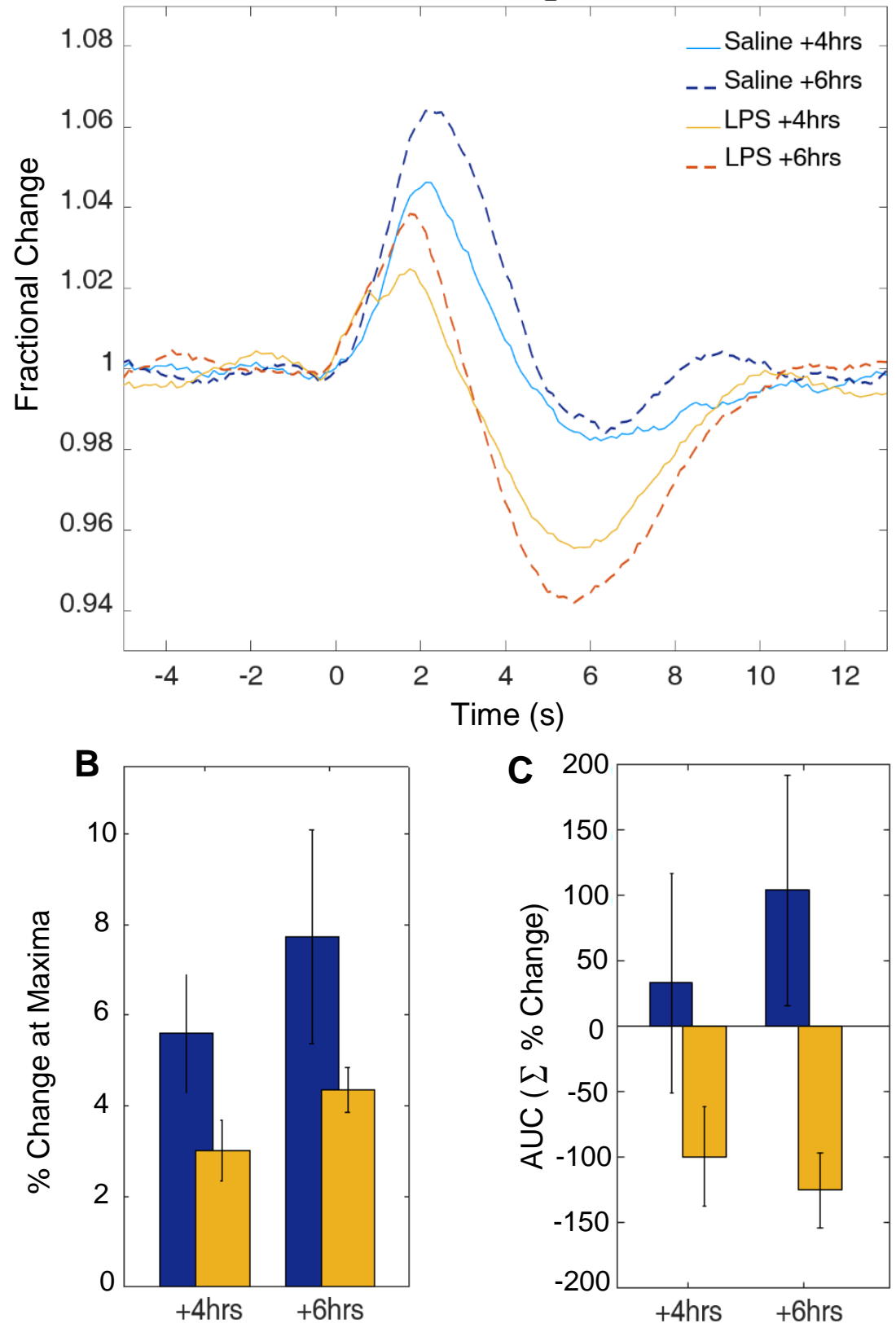

Saline $\square$ LPS 
bioRxiv preprint doi: https://doi.org/10.1101/498089; this version posted December 17, 2018. The copyright holder for this preprint (which was not certified by peer review) is the author/funder. All rights reserved. No reuse allowed without permission.

\section{Somatosensory Cortex (SS)}

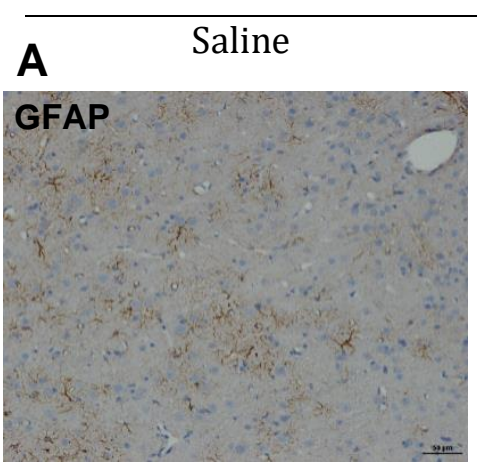

E

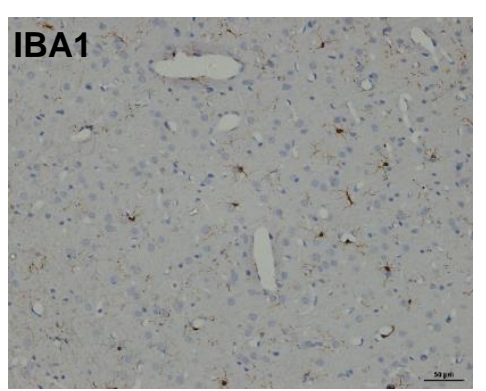

25

I

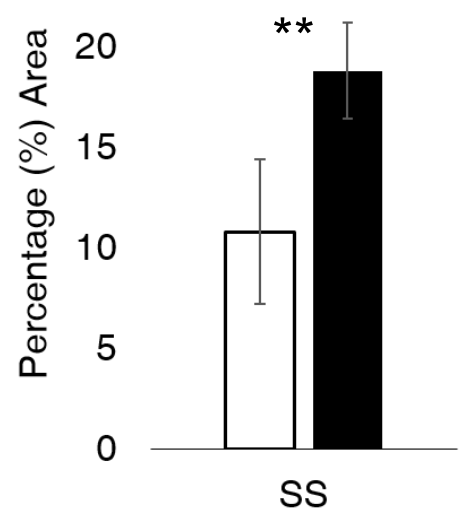

B

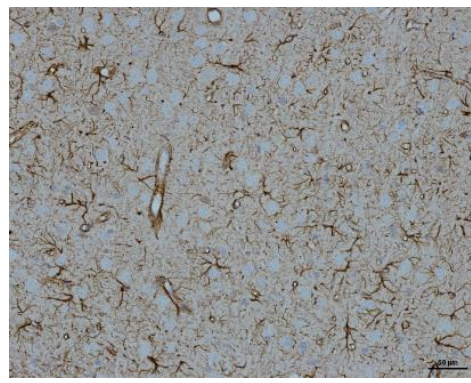

$\mathbf{F}$

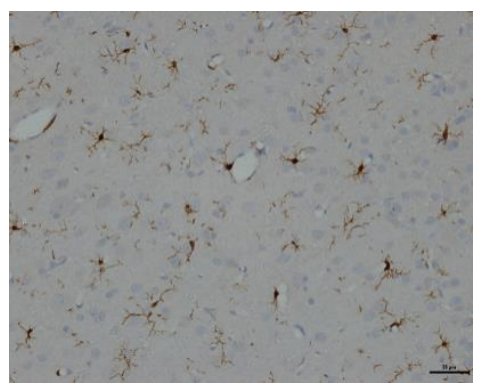

40

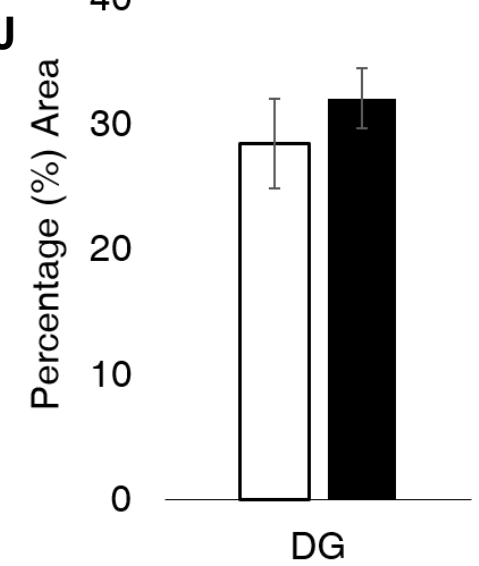

Hippocampus (Dentate Gyrus [DG])

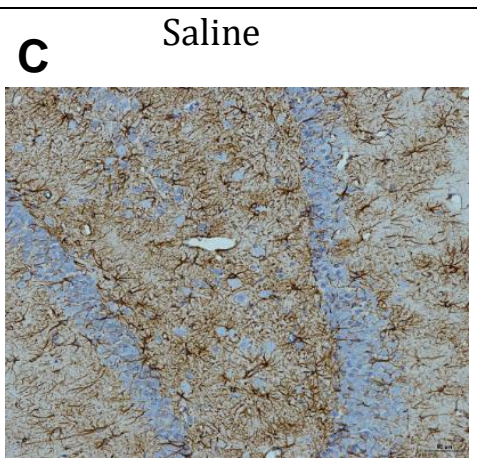

G

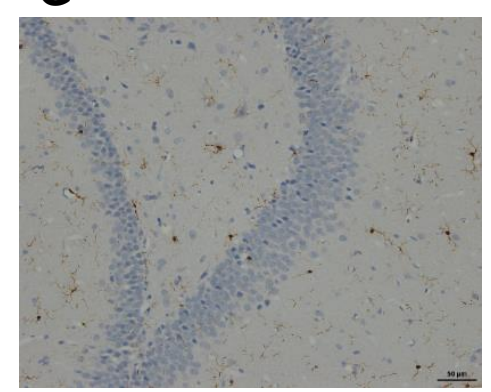

K

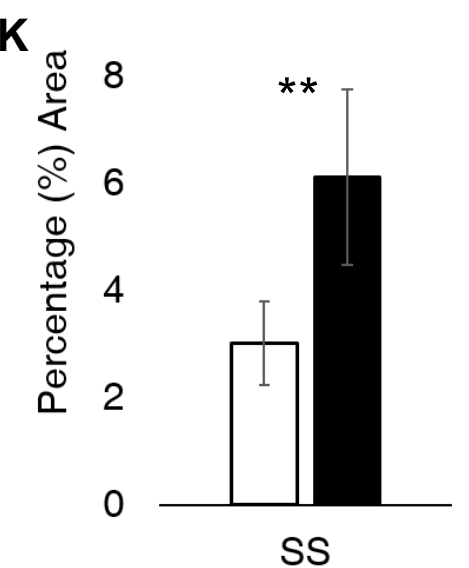

D

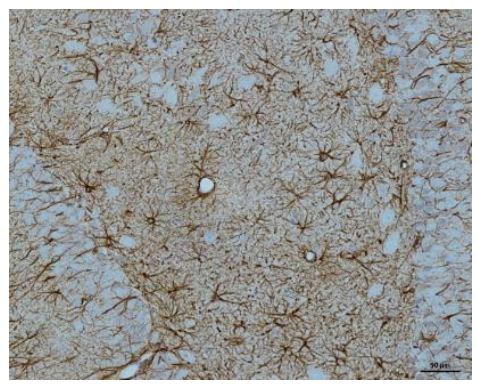

H

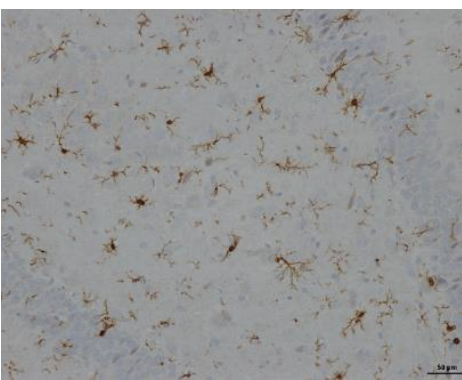

L 10

$\square$ Saline

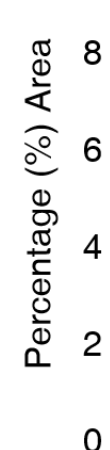

*

LPS

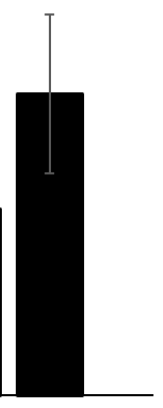

DG 
bioRxiv preprint doi: https://doi.org/10.1101/498089; this version posted December 17, 2018. The copyright holder for this preprint (which was not certified by peer review) is the author/funder. All rights reserved. No reuse allowed without permission.

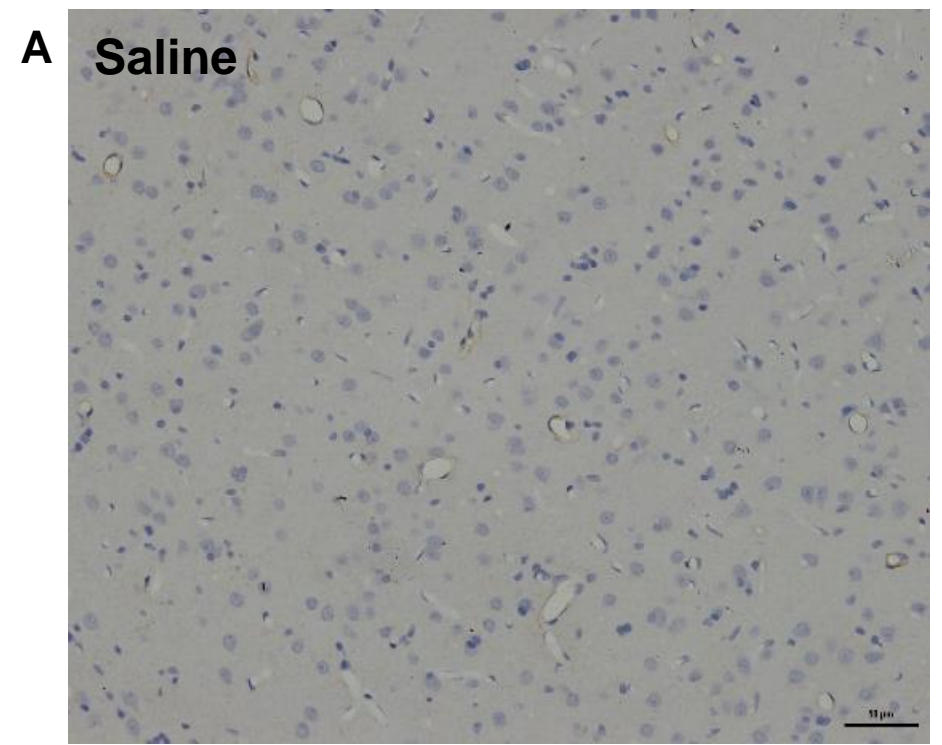

\section{B}

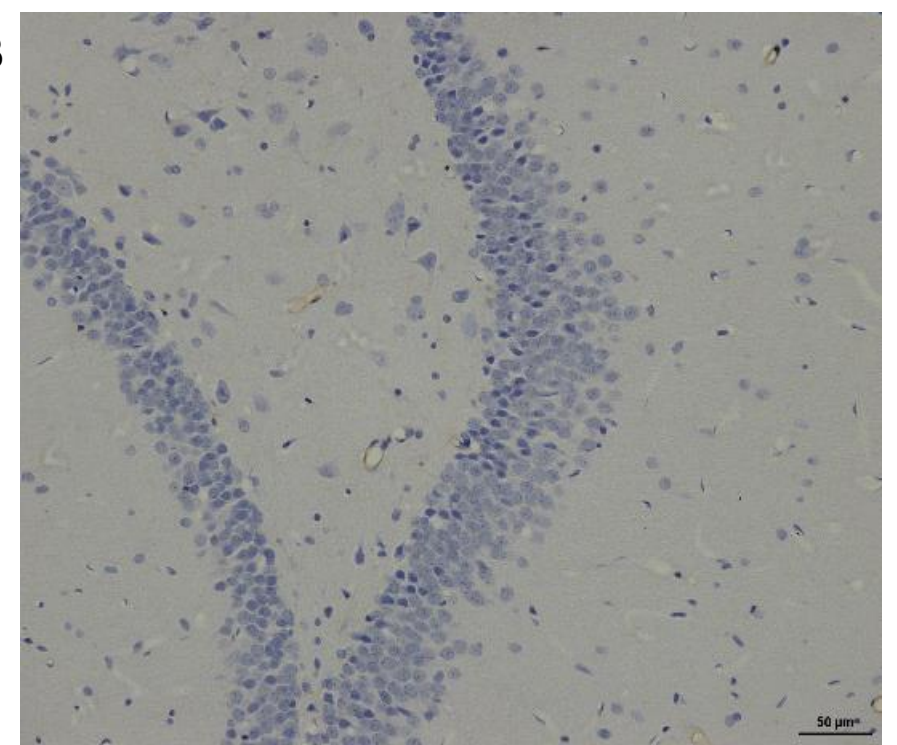

C LPS

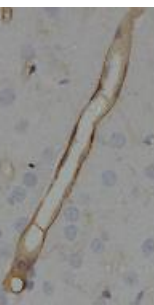

D

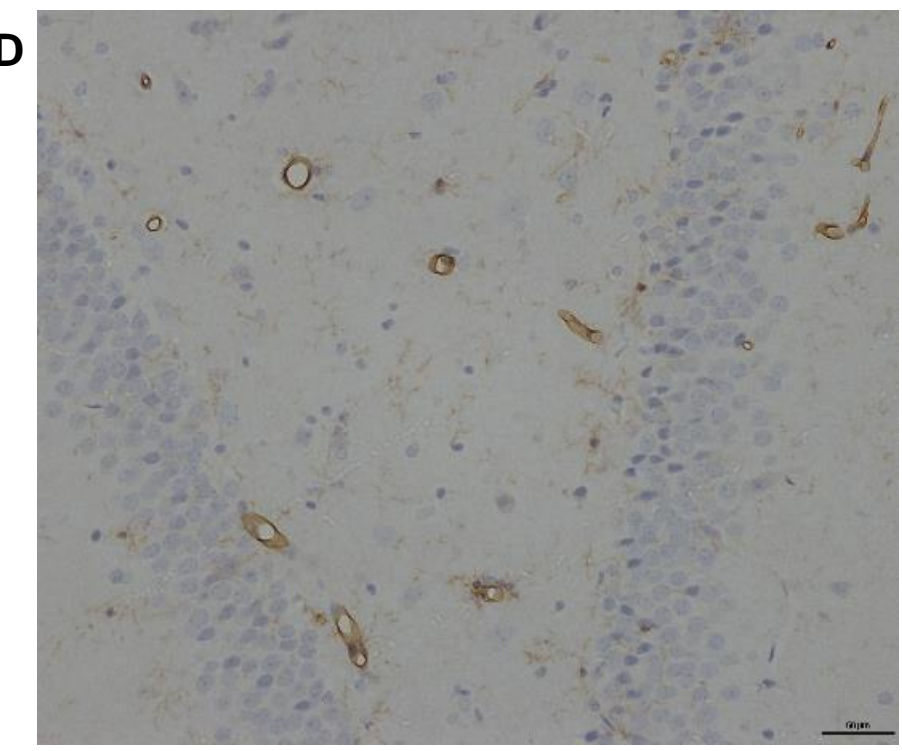

$\mathbf{E}$

**

F

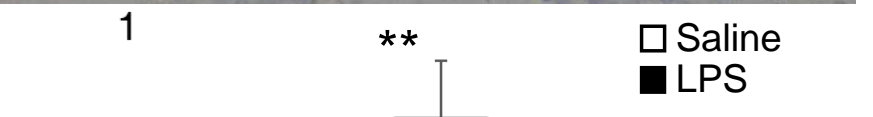

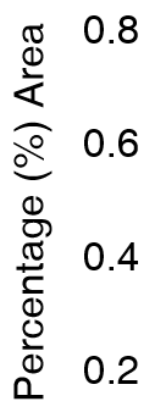

0

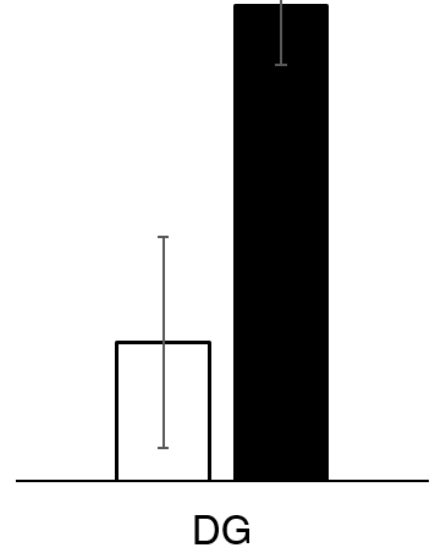

